\title{
Adaptive Autocentering Control for an Active Magnetic Bearing Supporting a Rotor with Unknown Mass Imbalance
}

\author{
Kai-Yew Lum, Vincent T. Coppola, and Dennis S. Bernstein, Member, IEEE
}

\begin{abstract}
This paper presents a new approach, called adaptive autocentering, that compensates for transmitted force due to imbalance in an active magnetic bearing system. Under the proposed control law, a rigid rotor achieves rotation about the mass center and principal axis of inertia. The basic principle of this approach is to perform on-line identification of the physical characteristics of rotor imbalance and to use the identification results to tune a stabilizing controller. This approach differs from the usual strategy of adaptive feedforward compensation, which models the effect of imbalance as an external disturbance or measurement noise, and then cancels this effect by generating a synchronous reference signal. Unlike adaptive feedforward compensation, adaptive autocentering control is frequency independent and works under varying rotor speed. Performance of the control algorithm is demonstrated in simulation examples for the case of rigid rotors with static or dynamic imbalance.
\end{abstract}

\section{INTRODUCTION}

$\mathbf{R}$ OTOR imbalance in active magnetic bearing (AMB) systems is manifested as synchronous rotor displacement as well as synchronous transmitted force. Recent approaches to imbalance compensation with AMB fall into the category of adaptive feedforward compensation (AFFC). In such approaches, the undesired vibration due to imbalance is modeled as an external disturbance in the case of compensation for displacement, or as measurement noise in the case of compensation for transmitted force. Whereas AFFC methods are based on cancellation of these vibration signals by feedforward injection of a synchronous reference signal into the control loop, they differ mainly in the adaptation schemes employed to generate the reference signal. Recent examples include modelbased observer design [5], [16], [17], notch filter design [12], least-squares optimization [10], [11], and others [18]. These methods are frequency dependent because the synchronous reference signal generator has parameters that are functions of the rotor spin rate, thus requiring continual adaptation as the spin rate varies. This frequency dependence is purely a consequence of the modeling approach since, as we know from physics, rotor imbalance is independent of the spin rate, and particularly so in the case of rigid rotors. To overcome the problem of frequency dependence, certain AFFC algorithms employ look-up tables [11] while others include recursive

Manuscript received October 25, 1995; revised February 20, 1996. This work was supported in part by the Air Force Office of Scientific Research under Grant F49620-95-1-0019.

The authors are with the Department of Aerospace Engineering, University of Michigan, Ann Arbor, MI 48109-2118 USA.

Publisher Item Identifier S 1063-6536(96)06623-7. adaptation or frequency tracking schemes [18]. The main difficulty of such schemes is that when the spin rate is rapidly varying or when the rotor crosses the critical speed, poor performance is generally observed.

We propose an alternative approach to imbalance compensation with AMB based on the notion that physical imbalance is independent of the spin rate. This approach consists of on-line identification of physical imbalance parameters, that is, the location of the mass center and the direction of the principal axis of inertia. These identified parameters are used to update a stabilizing controller for the AMB closed loop. The result is a feedback stabilizing control scheme that guarantees closed-loop stability by continuous parameter update. The main advantage of this approach is that once identification is achieved, adaptation can be halted and the identified parameters stored in memory. The same values can then be used at any spin rate, during spin-up or in the neighborhood of the critical speed determined by the stiffness of the suspension.

The above idea, which was proposed in [13 and [14], is known as adaptive virtual autobalancing (AVA) control. AVA was inspired by the mechanical properties of a passive device, namely, the autobalancer [1], [8], [21]. In the case of a rigid rotor with dynamic imbalance, AVA was shown to achieve imbalance identification and rotation about the geometric axis.

In the present paper, we develop adaptive autocentering (AAC) control. Like AVA, the objective of AAC control is to identify the imbalance of a rigid rotor; unlike AVA, however, AAC aims to achieve rotation about the mass center and principal axis of inertia instead of the geometric axis. A more comprehensive title for AAC would be "inertial autocentering" as opposed to "geometric autocentering." However, for simplicity, the word "inertial" is omitted hereafter. We shall show that the AAC control objective is equivalent to the attenuation of synchronous rotor vibration caused by mass imbalance. Section II outlines the linear AMR model with constant stiffness coefficients that will be assumed in the derivation of the AAC controller. In Section III, we consider the planar motion of a statically unbalanced, AMB-mounted rotor with known mass and rotating at a constant spin rate. By assuming that the rotor mass center location is known, we derive a stabilizing controller that achieves rotation about the mass center. In Section IV, we consider the general case in which the mass center is unknown, and formally introduce AAC control as an adaptive implementation of the stabilizing 
controller derived for known mass center location. In addition, we show that for planar motion the AAC closed-loop dynamics can be transformed into a set of linear, time-invariant differential equations. Convergence of the AAC closed loop is then equivalent, according to Floquet theory, to the stability of the equivalent linear time-invariant system, which is established in Section V using root locus arguments. Conditions that guarantee stability robustness with respect to small variations in the spin rate are also derived. The performance of AAC control for static imbalance is demonstrated by means of a simulation example in Section VI. Section VII discusses implementation considerations that exploit the unique features of this approach. AAC control is extended in Section VIII to the case of a threedimensional, dynamically unbalanced rotor by considering its implementation in two planes, assuming again a linear AMB model. Results in this case are demonstrated in simulation.

\section{Modeling OF A CURRENT-BiAsed Radial ACTIVE MagnetiC BEARING}

A simplified model of an eight-pole, current biased radial active magnetic bearing ( $\mathrm{AMB}$ ) is represented in Fig. 1, where four electromagnets are shown to be powered by the bias current $i_{0}$ and respective control currents $\pm i_{x}$ and $\pm i_{y}$. Assuming lossless ferrous material and neglecting flux leakages, the relationships between the magnetic forces $F_{x}$ and $F_{y}$ acting on the rotor on the one hand, and the coil currents and the displacement of the rotor on the other can be obtained through derivation of the energy stored in the air gaps as

$$
\begin{aligned}
& F_{x}=k\left[\left(\frac{i_{0}+i_{x}}{h_{0}-X \cos \alpha}\right)^{2}-\left(\frac{i_{0}-i_{x}}{h_{0}+X \cos \alpha}\right)^{2}\right] \\
& F_{y}=k\left[\left(\frac{i_{0}+i_{y}}{h_{0}-Y \cos \alpha}\right)^{2}-\left(\frac{i_{0}-i_{y}}{h_{0}+Y \cos \alpha}\right)^{2}\right]
\end{aligned}
$$

where $k=(1 / 4) N^{2} A \mu_{0} \quad \cos \alpha, N$ is the number of turns in each coil, $A$ is the projected area of each air gap, $\mu_{0}$ is the permeability of air, $h_{0}$ is the nominal air gap, $(X, Y)$ are the Cartesian coordinates of the rotor center relative to the bearing center, and $\alpha$ is half the angle between the poles of an electromagnet. Moreover, assuming small rotor displacement and small control currents, and thus taking the Taylor's series expansions of (1) and (2), the magnetic forces are given by the well-known linear AMB equations

$$
\begin{aligned}
F_{x}\left(X, i_{x}\right) & =K_{h} X+K_{i} i_{x} \\
F_{y}\left(Y, i_{y}\right) & =K_{h} Y+K_{i} i_{y}
\end{aligned}
$$

where the position stiffness $K_{h}$ and current stiffness $K_{i}$ can be shown to be given by $K_{h}=4 k\left(i_{0}^{2} / h_{0}^{3}\right) \cos \alpha$ and $K_{i}=4 k i_{0} / h_{0}^{2}$. See, for example, [19] and references therein on design and modeling of AMB. Other references on AMB modeling include [6], [7], and [15].

\section{Autocentering Control for Planar ROTOR WITH KNOWN STATIC IMBALANCE}

Now consider the planar, statically unbalanced rotor shown in Fig. 2. The mass center $\mathrm{C}$ of the rotor is located in an

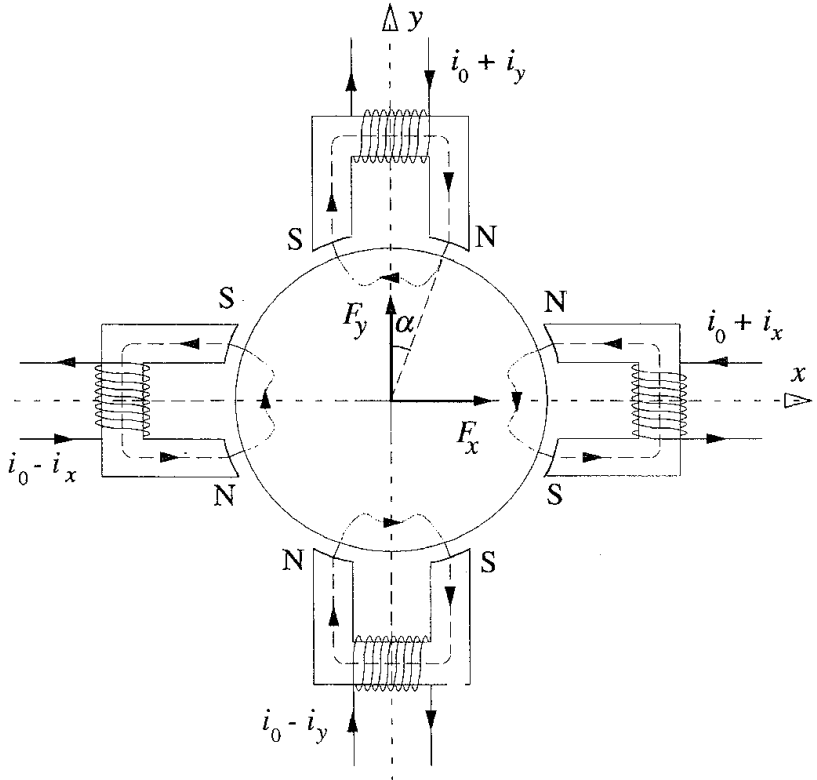

Fig. 1. Current biased radial magnetic bearing.

arbitrary body frame with origin $\mathrm{O}$ and Cartesian coordinates $(\xi, \zeta)$. Let $\mathrm{N}$ denote the bearing center and let $(x, y)$ denote the Cartesian coordinates of $\mathrm{C}$ in the inertial frame with $\mathrm{N}$ as the origin. We assume henceforth that the rotor is spinning at a constant spin rate $\Omega$. Moreover, suppose that gravity is acting in the negative- $y$ direction. It then follows from (3) and (4) that the planar motion of the rotor acted upon by the AMB and gravity is given by Newton's second law as

$$
\begin{aligned}
& \ddot{x}=\frac{K_{h}}{m}(x-\xi \cos \Omega t+\zeta \sin \Omega t)+\frac{K_{i}}{m} i_{x} \\
& \ddot{y}=\frac{K_{h}}{m}(y-\xi \sin \Omega t-\zeta \cos \Omega t)+\frac{K_{i}}{m} i_{y}-g
\end{aligned}
$$

where $m$ is the mass of the rotor, and where we have used the identities

$$
\begin{aligned}
& X=x-\xi \cos \Omega t+\zeta \sin \Omega t \\
& Y=y-\xi \sin \Omega t-\zeta \cos \Omega t
\end{aligned}
$$

Note that if $i_{x}=i_{y}=0$, then the rotor motion in both the $x$ - and $y$-directions is a simple harmonic oscillator with positive stiffness coefficient $K_{h} / m$ and subject to sinusoidal excitations. Obviously, the equilibrium point $(0,0)$ is unstable in the absence of control.

To achieve rotation about the mass center, a control law for $i_{x}$ and $i_{y}$ must be chosen so that both $x$ and $y$ converge asymptotically to zero. For this purpose, consider the proportional-differential control with constant bias given by

$$
\begin{aligned}
i_{x} & =-\frac{K_{h}}{K_{i}} X-\frac{m}{K_{i}}\left(k_{1} \dot{x}+k_{2} x\right) \\
i_{y} & =-\frac{K_{h}}{K_{i}} Y-\frac{m}{K_{i}}\left(k_{1} \dot{y}+k_{2} y\right)+\frac{m g}{K_{i}}
\end{aligned}
$$

where $k_{1}$ and $k_{2}$ are positive constants to be chosen. Note that implementation of (8) and (9) requires knowledge of the stiffness coefficients $K_{h}$ and $K_{i}$ and the mass $m$ of the rotor, 


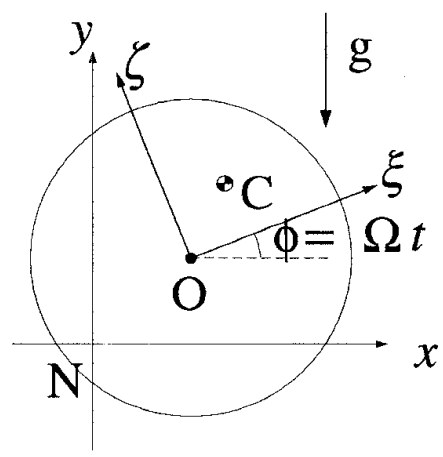

Fig. 2. Planar rotor with static imbalance.

which we shall assume hereafter. Substituting (7)-(9) into (5) and (6) yields the equations of motion of the closed-loop system

$$
\begin{aligned}
& \ddot{x}=-k_{1} \dot{x}-k_{2} x \\
& \ddot{y}=-k_{1} \dot{y}-k_{2} y .
\end{aligned}
$$

Thus, the control law given by (8) and (9) asymptotically stabilizes the motion of the mass center at the origin. Consequently, the rotor achieves rotation about the mass center.

An important property of rotation about the mass center is that the steady-state forces exerted by the AMB on the rotor have zero synchronous components. Indeed, setting $x, y, \dot{x}$ and $\dot{y}$ to zero in (8) and (9) yields ${ }_{x}=-K_{h} X / K_{i}$ and $i_{y}=\left(-K_{h} Y+m g\right) / K_{i}$ which, when substituted into (3) and (4), give $F_{x} \equiv 0$ and $F_{y} \equiv m g$. Thus, the only actuation force required at steady state is that which supports the rotor's weight. Achieving rotation about the mass center is thus equivalent to achieving vibration attenuation at the supports of the AMB system. Furthermore, at steady state, $X=-\xi \cos \Omega t+\zeta \sin \Omega t$ and $Y=-\xi \sin \Omega t-\zeta \cos \Omega t$, that is, $\mathrm{C}$ coincides with $\mathrm{N}$ and the geometric center $\mathrm{O}$ describes a circle of radius $\sqrt{\xi^{2}+\zeta^{2}}$ centered at $\mathrm{N}$.

Equations (8) and (9) provide a simple feedback control law that asymptotically stabilizes the AMB rotor dynamics (5) and (6). In practice, however, rotor imbalance is not precisely known. As a result, the mass center position $(x, y)$ and velocity $(\dot{x}, \dot{y})$ are unknowns, which renders the implementation of (8) and (9) impossible. Estimates of these variables are therefore required, and our goal is to provide these estimates by incorporating an adaptation algorithm that effectively identifies the imbalance parameters $(\xi, \zeta)$. This algorithm is discussed in the following section.

\section{AdAPtive Autocentering CONTROL FOR UNKNOWN STATIC IMBALANCE}

In the previous section, the feedback control law (8) and (9) asymptotically stabilizes the AMB rotor dynamics (5) and (6). However, to implement this control law, measurements of the mass center position $(x, y)$ and velocity $(\dot{x}, \dot{y})$ are required. Whereas the geometric center position $(X, Y)$ can usually be measured using displacement sensors such as eddy-current or capacitive sensors, and the rotation angle $\phi=\Omega t$ can be measured using optical encoders or Hall effect sensors, the imbalance parameters $(\xi, \zeta)$ are in general unknown, and thus $(x, y)$ and $(\dot{x}, \dot{y})$ cannot be directly measured or computed. Our objective is thus to obtain on-line estimates of the rotor imbalance parameters $(\xi, \zeta)$ and use these estimates to compute the mass center position and velocity so that $(8)$ and (9) are implemented based on the computed values.

Consider the dynamical equations

$$
\begin{aligned}
& \ddot{\hat{\xi}}=-k_{3} \dot{\hat{\xi}}-k_{4}(\hat{x} \cos \Omega t+\hat{y} \sin \Omega t) \\
& \ddot{\hat{\zeta}}=-k_{3} \dot{\hat{\zeta}}-k_{5}(-\hat{x} \sin \Omega t+\hat{y} \cos \Omega t)
\end{aligned}
$$

where $(\hat{x}, \hat{y})$ are defined by

$$
\begin{aligned}
& \hat{x} \triangleq X+\hat{\xi} \cos \Omega t-\hat{\zeta} \sin \Omega t \\
& \hat{y} \triangleq Y+\hat{\xi} \sin \Omega t+\hat{\zeta} \cos \Omega t
\end{aligned}
$$

and $k_{3}>0, k_{4} \in \Re$ and $k_{5} \in \Re$ are constants to be chosen. Next, define

$$
\begin{aligned}
& \hat{\dot{x}} \triangleq \dot{X}-\hat{\xi} \Omega \sin \Omega t-\hat{\zeta} \Omega \cos \Omega t \\
& \hat{\dot{y}} \triangleq \dot{Y}+\hat{\xi} \Omega \cos \Omega t-\hat{\zeta} \Omega \sin \Omega t
\end{aligned}
$$

Finally, instead of (8) and (9), let the control currents be given by

$$
\begin{aligned}
& i_{x}=-\frac{K_{h}}{K_{i}} X-\frac{m}{K_{i}}\left(k_{1} \hat{\dot{x}}+k_{2} \hat{x}\right) \\
& i_{y}=-\frac{K_{h}}{K_{i}} Y-\frac{m}{K_{i}}\left(k_{1} \hat{\dot{y}}+k_{2} \hat{y}\right)+\frac{m g}{K_{i}} .
\end{aligned}
$$

Equations (12)-(19) comprise the adaptive autocentering control law (AAC) for the rotor dynamics (5) and (6). A representation of the AAC closed loop is given in Fig. 3 where $Q_{1}(\Omega, t)$ and $Q_{2}(\Omega, t)$ are time-dependent matrices parameterized by $\Omega$ and given by

$$
\begin{aligned}
Q_{1}(\Omega, t) & =\left[\begin{array}{cccc}
\cos \Omega t & \sin \Omega t & 0 & 0 \\
-\sin \Omega t & \cos \Omega t & 0 & 0
\end{array}\right] \\
Q_{2}(\Omega, t) & =\left[\begin{array}{cc}
\cos \Omega t & -\sin \Omega t \\
\sin \Omega t & \cos \Omega t \\
-\Omega \sin \Omega t & -\Omega \cos \Omega t \\
\Omega \cos \Omega t & -\Omega \sin \Omega t
\end{array}\right] .
\end{aligned}
$$

We can view the loop that involves $Q_{1}(\Omega, t), Q_{2}(\Omega, t)$, and (4.1) and (4.2) or, equivalently, (12)-(17), as an adaptation loop that updates the variables $(\hat{\xi}, \hat{\zeta})$ which we shall show in the following sections to be asymptotic estimates of the imbalance parameters $(\xi, \zeta)$. Note that implementation of (12)-(19) requires measurements of the translational position $(X, Y)$ and velocity $(\dot{X}, \dot{Y})$ of the rotor geometric center as well as the angular velocity $\Omega$, all of which can be obtained in practice. ${ }^{1}$

The equations of motion of the closed-loop system comprised of the rotor dynamics (5)-(7) and AAC control law (18) and (19) are given by

$$
\begin{aligned}
& \ddot{x}=-k_{1} \hat{\dot{x}}-k_{2} \hat{x} \\
& \ddot{y}=-k_{1} \hat{\dot{y}}-k_{2} \hat{y} .
\end{aligned}
$$

${ }^{1}$ In the case where velocity measurements are not available, additional schemes such as state observer or taking derivatives of position measurements can be envisioned. Analysis of the effects of errors thus introduced is beyond the scope of this paper. 


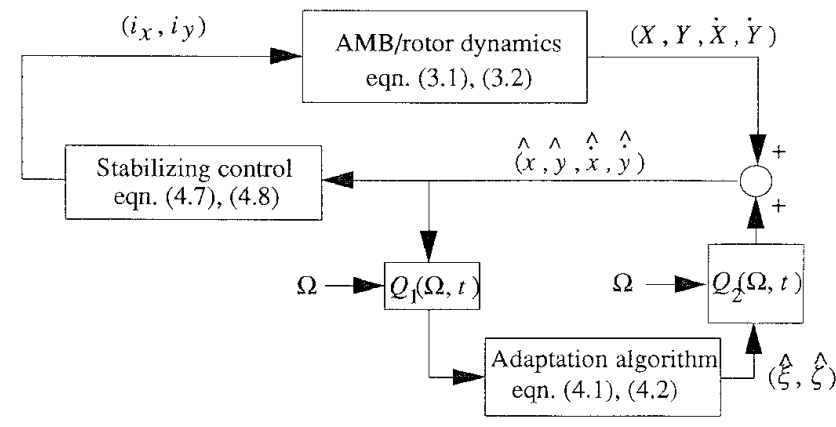

Fig. 3. AAC closed-loop block diagram.

Next, define $\tilde{\xi} \triangleq \hat{\xi}-\xi$ and $\tilde{\zeta} \triangleq \hat{\zeta}-\zeta$. Then, substituting (14) $-(17)$ and (7) into (20) and (21), and replacing $\hat{\xi}$ and $\hat{\zeta}$ by, respectively, $\tilde{\xi}+\xi$ and $\tilde{\zeta}+\zeta$ in (12) and (13), the closed-loop system becomes

$$
\begin{aligned}
\ddot{x}= & -k_{1} \dot{x}-k_{2} x+k_{1} \Omega(\tilde{\xi} \sin \Omega t+\tilde{\zeta} \cos \Omega t) \\
& -k_{2}(\tilde{\xi} \cos \Omega t-\tilde{\zeta} \sin \Omega t) \\
\ddot{y}= & -k_{1} \dot{y}-k_{2} y-k_{1} \Omega(\tilde{\xi} \cos \Omega t-\tilde{\zeta} \sin \Omega t) \\
& -k_{2}(\tilde{\xi} \sin \Omega t+\tilde{\zeta} \cos \Omega t) \\
\ddot{\tilde{\xi}}= & -k_{3} \tilde{\tilde{\xi}}-k_{4} \tilde{\xi}-k_{4}(x \cos \Omega t+y \sin \Omega t) \\
\ddot{\tilde{\zeta}}= & -k_{3} \dot{\tilde{\zeta}}-k_{5} \tilde{\zeta}-k_{5}(-x \sin \Omega t+y \cos \Omega t) .
\end{aligned}
$$

It can easily be seen that (22)-(25) can be rewritten as a system of eight linear differential equations of the form

$$
\dot{Z}=A(\Omega, t) Z
$$

where $Z=(x, y, \dot{x}, \dot{y}, \tilde{\xi}, \tilde{\zeta}, \dot{\tilde{\xi}}, \dot{\tilde{\zeta}})$, and $A(\Omega, t)$ is a timedependent, periodic $8 \times 8$ matrix with period $2 \pi / \Omega$. If (26) is exponentially stable, then the motion of the mass center is stabilized. Moreover, $(\tilde{\xi}, \tilde{\zeta})$ then converges to zero and thus $(\hat{\xi}, \hat{\zeta})$ are asymptotic estimates of $(\xi, \zeta)$.

The following theorem is a well-known result. The proof can be found in [4].
Theorem 1 (Floquet): Consider the $n$th order linear system $\dot{Z}=A(t) Z, A(t+T)=A(t)$ for all $t, T>0$. Then every fundamental matrix solution $\Phi(t)$ of this system has the form $\Phi(t)=P(t) e^{\tilde{A} t}$ where $P(t)$ is an $n \times n$ periodic matrix with period $T$ and $\tilde{A}$ is an $n \times n$ constant matrix. Moreover, $P(t)$ is nonsingular for all $t$ and the transformation $z=P(t) Z$ transforms the system into the linear time-invariant system $\dot{z}=\tilde{A} z$. In addition, the origin of the system $\dot{Z}=A(t) Z$ is exponentially stable if and only if $\tilde{A}$ is Hurwitz.

Consider now the change of variables $z=P(\Omega, t) Z$, where $P(\Omega, t)$ is defined by the equation shown at the bottom of the page in sparse matrix representation. It can easily be verified that $P(\Omega, t)$ transforms (26) into the linear time-invariant system

$$
\dot{z}=\tilde{A}(\Omega) z, \quad z \in \Re^{8}
$$

where $\tilde{A}(\Omega)$ is defined by the equation shown at the bottom of the page. Hence, $P(\Omega, t)$ and $\tilde{A}(\Omega)$ satisfy the conditions of Theorem 1 for the system (26). Consequently, convergence of the AAC algorithm can be established by investigating the stability of $\tilde{A}(\Omega)$ as a function of $k_{1}, \cdots, k_{5}$. We can in theory apply the Routh-Hurwitz criterion to the characteristic polynomial of $\tilde{A}(\Omega)$. However, this approach is intractable due to the high dimensionality. A root locus approach is therefore adopted.

\section{Convergence Proof by Root locus Analysis}

Let $\Omega$ be nonzero and $\left(k_{1}, k_{2}, k_{3}\right)$ be arbitrary positive numbers. We shall now show by root locus analysis that a pair of numbers $\left(k_{4}, k_{5}\right)$ can be found such that the closed loop (22)-(25) is asymptotically stable. First, we rewrite $\tilde{A}(\Omega)$ in the form $\tilde{A}(\Omega)=A(\Omega)-B_{1} k_{4} C_{1}-B_{2} k_{5} C_{2}$, where $A(\Omega)$ is defined by the equation shown at the bottom of the next page. $B_{1}=\left[\begin{array}{llllllll}0 & 0 & 0 & 0 & 0 & 0 & 1 & 0\end{array}\right]^{\mathrm{T}}, B_{2}=\left[\begin{array}{llllllll}0 & 0 & 0 & 0 & 0 & 0 & 0 & 1\end{array}\right]^{\mathrm{T}}, C_{1}=$ $\left[\begin{array}{llllllll}1 & 0 & 0 & 0 & 1 & 0 & 0 & 0\end{array}\right]$ and $C_{2}=\left[\begin{array}{llllllll}0 & 1 & 0 & 0 & 0 & 1 & 0 & 0\end{array}\right]$. Moreover, let $B \triangleq\left[B_{1} B_{2}\right]$ and $C \triangleq\left[C_{1}^{\mathrm{T}} C_{2}^{\mathrm{T}}\right]^{\mathrm{T}}$. It can be verified that

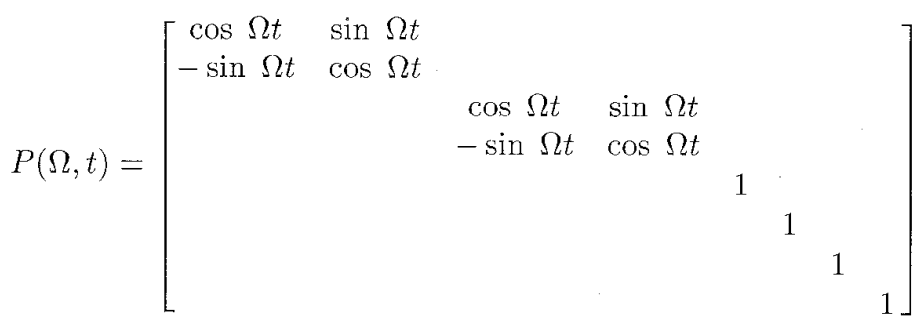

$$
\begin{aligned}
& \tilde{A}(\Omega)=\left[\begin{array}{cccccccc}
0 & \Omega & 1 & 0 & 0 & 0 & 0 & 0 \\
-\Omega & 0 & 0 & 1 & 0 & 0 & 0 & 0 \\
-k_{2} & 0 & -k_{1} & \Omega & -k_{2} & k_{1} \Omega & 0 & 0 \\
0 & -k_{2} & -\Omega & -k_{1} & -k_{1} \Omega & -k_{2} & 0 & 0 \\
0 & 0 & 0 & 0 & 0 & 0 & 1 & 0 \\
0 & 0 & 0 & 0 & 0 & 0 & 0 & 1 \\
-k_{4} & 0 & 0 & 0 & -k_{4} & 0 & -k_{3} & 0 \\
0 & -k_{5} & 0 & 0 & 0 & -k_{5} & 0 & -k_{3}
\end{array}\right]
\end{aligned}
$$


$(A(\Omega), B, C)$ is a realization of the $2 \times 2$ transfer matrix

$$
\begin{aligned}
G(s) & =\left[\begin{array}{cc}
G_{1}(s) & G_{2}(s) \\
-G_{2}(s) & G_{1}(s)
\end{array}\right] \\
G_{1}(s) & =\frac{N_{1}(s)}{D(s)}, \quad G_{2}(s)=\frac{N_{2}(s)}{D(s)}
\end{aligned}
$$

where $G_{1}(s)$ and $G_{2}(s)$ have the common denominator

$$
\begin{aligned}
D(s)= & s\left(s+k_{3}\right)\left[s^{4}+2 k_{1} s^{3}+\left(k_{1}^{2}+2 k_{2}+2 \Omega^{2}\right) s^{2}\right. \\
& \left.+2 k_{1}\left(\Omega^{2}+k_{2}\right) s+\Omega^{4}+\left(k_{1}^{2}-2 k_{2}\right) \Omega^{2}+k_{2}^{2}\right]
\end{aligned}
$$

and respective numerators

$N_{1}(s)=s^{4}+2 k_{1} s^{3}+\left(k_{1}^{2}+k_{2}+2 \Omega^{2}\right) s^{2}+k_{1} k_{2} s+\left(\Omega^{2}-k_{2}\right) \Omega^{2}$

and

$$
N_{2}(s)=k_{1} \Omega s^{2}+\left(k_{1}^{2}-2 k_{2}\right) \Omega s-k_{1} \Omega^{3} .
$$

Note that the quartic factor of $D(s)$ is the characteristic polynomial of the leading $4 \times 4$ principal submatrix of $A(\Omega)$ which has the four distinct roots

$$
\lambda_{1, \cdots, 4}=-\frac{1}{2} k_{1} \pm \frac{1}{2} \sqrt{k_{1}^{2}-4 k_{2}} \pm j \Omega .
$$

Since $A(\Omega)$ is upper triangular, these roots are eigenvalues of $A(\Omega)$, and they lie in the open left-half complex plane (OLHP). In addition, $A(\Omega)$ is not cyclic and has double eigenvalues $\lambda=-k_{3}$ and $\lambda=0$ which are semisimple. Hence, these eigenvalues are not repeated in $D(s)$.

For sufficiently small values of $k_{4}$ and $k_{5}$, the stability of (27) then depends on the loci of the double eigenvalue $\lambda=0$. We shall therefore analyze the root locus of $\left(A(\Omega), B_{1}, C_{1}\right)$ and that of $\left(A(\Omega)-B_{1} k_{4} C_{1}, B_{2}, C_{2}\right)$ for fixed values of $k_{4}{ }^{2}$. First, note that $\left(A(\Omega), B_{1}, C_{1}\right)$ is a realization of the transfer function $G_{1}(s)$. Next, for all $k_{4},\left(A(\Omega)-B_{1} k_{4} C_{1}, B_{2}, C_{2}\right)$ is a realization of the transfer function

$$
\begin{aligned}
& G_{3}\left(s, k_{4}\right) \\
& =\frac{k_{4} G_{2}^{2}(s)}{1+k_{4} G_{1}(s)}+G_{1}(s) \\
& =\frac{s\left(s+k_{3}\right) N_{1}(s)}{s\left(s+k_{3}\right)\left[D(s)+k_{4} N_{1}(s)\right]} \\
& \quad+\frac{k_{4}\left[s^{4}+2 k_{1} s^{3}+\left(2 \Omega^{2}+k_{1}^{2}\right) s^{2}-2 k_{1} \Omega^{2} s+\Omega^{4}\right]}{s\left(s+k_{3}\right)\left[D(s)+k_{4} N_{1}(s)\right]} .
\end{aligned}
$$

The following is a well-known fact [3].

${ }^{2}$ Due to the antisymmetry of $G(s)$, this is equivalent to analyzing the root locus of $\left(A(\Omega), B_{2}, C_{2}\right)$ and that of $\left(A(\Omega)-B_{2} k_{5} C_{2}, B_{1}, C_{1}\right)$ for fixed values of $k$.
Lemma 1: For the SISO transfer function $h(s)$, consider the feedback closed-loop transfer function $H(s)=h(s) / 1+$ $k h(s)$. Then, if $k>0$ (respectively, $k<0$ ), the portion of the real axis to the left of an odd (respectively, even) number of poles and zeros belongs to the root locus.

We shall now distinguish two cases.

\section{A. Case 1: $\Omega^{2}>k_{2}$}

In this case, the $s^{0}$ coefficient of $N_{1}(s)$ (that is, the product of the roots) is positive and, hence, $G_{1}(s)$ has an even number of ORHP zeros. However, $G_{1}(s)$ cannot have more than two such zeros, since the $s^{3}$ coefficient of the fourth-order polynomial $N_{1}(s)$ (that is, the negative sum of the roots) equals $2 k_{1}$ and hence is positive.

It thus follows from Lemma 1 that for $k_{4}>0$, the pole $\lambda=0$ moves into the OLHP, possibly returning to the ORHP for sufficiently large values of $k_{4}$. In other words, for sufficiently small positive values of $k_{4}$, the roots of $D(s)+k_{4} N_{1}(s)$ lie in the OLHP.

Now let $k_{4}$ be such that the roots of $D(s)+k_{4} N_{1}(s)$ lie in the OLHP. The stability of (27) then depends on the locus of the pole $\lambda=0$ of $G_{3}\left(s, k_{4}\right)$, and therefore depends on the number of ORHP zeros of $G_{3}\left(s, k_{4}\right)$. From (28) it can be seen that the zeros of $G_{3}\left(s, k_{4}\right)$ fall on the root locus of the transfer function

$$
g(s) \triangleq \frac{s^{4}+2 k_{1} s^{3}+\left(2 \Omega^{2}+k_{1}^{2}\right) s^{2}-2 k_{1} \Omega^{2} s+\Omega^{4}}{s\left(s+k_{3}\right) N_{1}(s)}
$$

with feedback gain $k_{4}$. It can be verified by forming the Routh table of the fourth-order numerator of $g(s)$ that $g(s)$ has at least one ORHP zero. However, since the $s^{3}$ coefficient of the numerator is positive, not all the zeros of $g(s)$ lie in the ORHP. Moreover, the $s^{0}$ coefficient of the numerator is positive, so there must be an even number of ORHP zeros. Therefore $g(s)$ has exactly two zeros in the ORHP. This, and the already seen fact that $N_{1}(s)$ has an even number of ORHP roots, imply by Lemma 1 that the pole $\lambda=0$ of $g(s)$ moves into the OLHP for $k_{4}>0$. It is then clear that for sufficiently small positive values of $k_{4}, G_{3}\left(s, k_{4}\right)$ has the same even number of ORHP zeros as $G_{1}(s)$, and no ORHP poles. Thus, it again follows from Lemma 1 that the pole $\lambda=0$ of $G_{3}\left(s, k_{4}\right)$ moves into the OLHP for $k_{5}>0$.

Based on the above discussion, we conclude that in the case $\Omega^{2}>k_{2},(27)$ is asymptotically stable for all positive values of $k_{1}$ and $k_{3}$ and for all sufficiently small positive values of $k_{4}$ and $k_{5}$.

$$
A(\Omega)=\left[\begin{array}{cccccccc}
0 & \Omega & 1 & 0 & 0 & 0 & 0 & 0 \\
-\Omega & 0 & 0 & 1 & 0 & 0 & 0 & 0 \\
-k_{2} & 0 & -k_{1} & \Omega & -k_{2} & k_{1} \Omega & 0 & 0 \\
0 & -k_{2} & -\Omega & -k_{1} & -k_{1} \Omega & -k_{2} & 0 & 0 \\
0 & 0 & 0 & 0 & 0 & 0 & 1 & 0 \\
0 & 0 & 0 & 0 & 0 & 0 & 0 & 1 \\
0 & 0 & 0 & 0 & 0 & 0 & -k_{3} & 0 \\
0 & 0 & 0 & 0 & 0 & 0 & 0 & -k_{3}
\end{array}\right]
$$




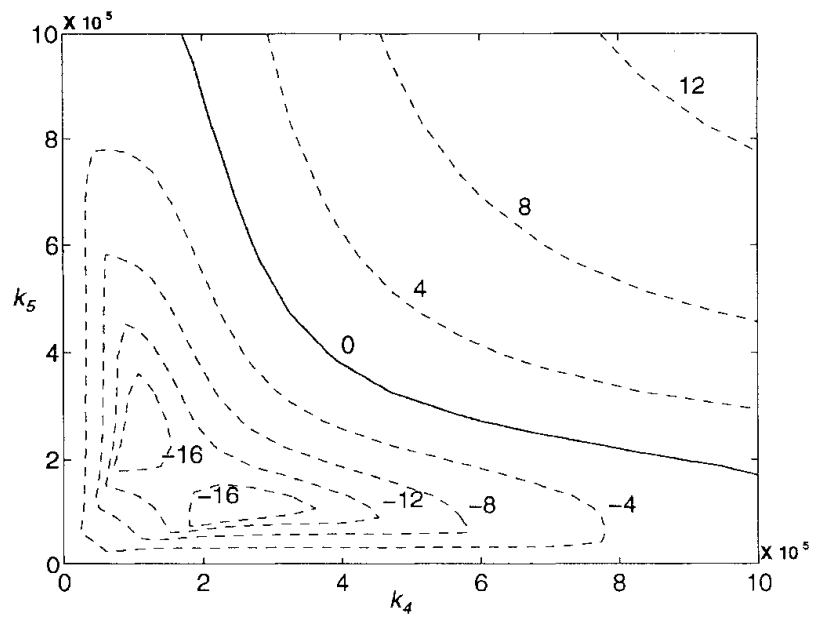

(a)

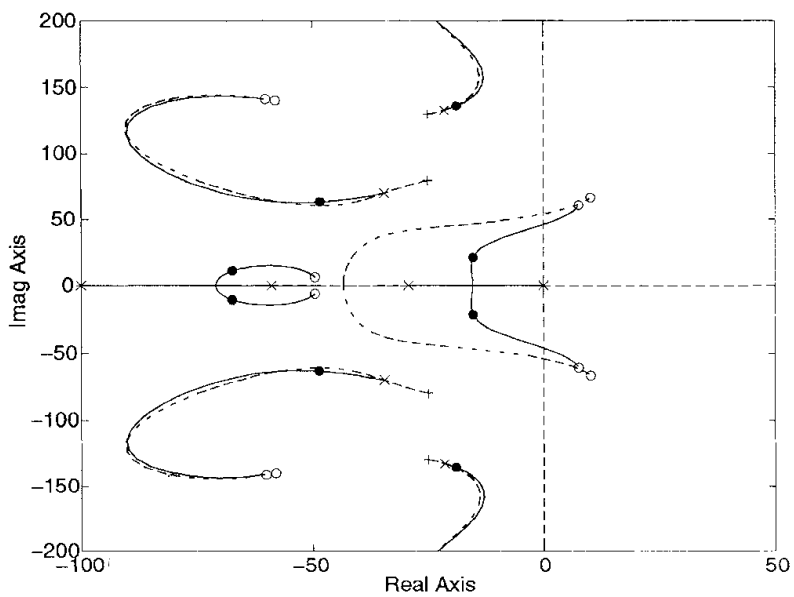

(b)

Fig. 4. Example 1: $\Omega^{2}>k_{2}$. (a) Contour plot of max. real parts of the closed-loop poles. (b) Root loci.

Example 1: Let $k_{1}=50 \mathrm{~s}^{-1}, k_{2}=1250 \mathrm{~s}^{-2}, k_{3}=$ $100 \mathrm{~s}^{-1}, \Omega=1000 \mathrm{r} / \mathrm{min}\left(104.7 \mathrm{rad}_{-} \mathrm{s}^{-1}\right)$, so that $\Omega^{2}>k_{2}$ is satisfied. Fig. 4(a) shows a contour plot of the maximum of the real parts of the closed-loop poles for $k_{4}>0$ and $k_{5}>0$. It can be seen in particular that $0<k_{4}<3500$ and $0<k_{5}<3500$ guarantee that all the closed-loop poles lie in the OLHP. In Fig. 4(b), the dotted lines represent the root locus of $G_{1}(s)$, and the solid lines represent the root locus of $G_{3}\left(s, k_{4}\right)$ for $k_{4}=1800 \mathrm{~s}^{-2}$. For instance, the closed-loop poles for $k_{5}=k_{4}=1800 \mathrm{~s}^{-2}$ are $-18.9 \pm 135.8 j,-49.0 \pm$ $63.1 j,-15.1 \pm 21.8 j$, and $-66.9 \pm 11.2 j$ (marked "•").

\section{B. Case 2: $\Omega^{2}<k_{2}$}

In this case, the $s^{0}$ coefficient of $N_{1}(s)$ is negative whereas the $s^{3}$ coefficient is still positive. Hence an odd number but not all of the zeros of $G_{1}(s)$ are in the ORHP. It follows from Lemma 1 that for sufficiently small negative values of $k_{4}$, the roots of $D(s)+k_{4} N_{1}(s)$ lie in the OLHP. Using an analogous argument as in Case 1, we can see that for sufficiently small negative values of $k_{4}, G_{3}\left(s, k_{4}\right)$ has an odd number of ORHP

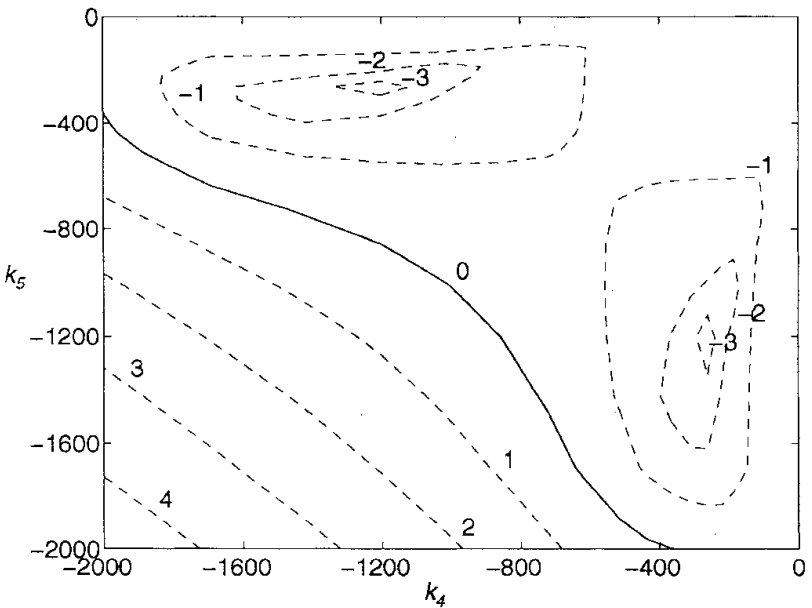

(a)

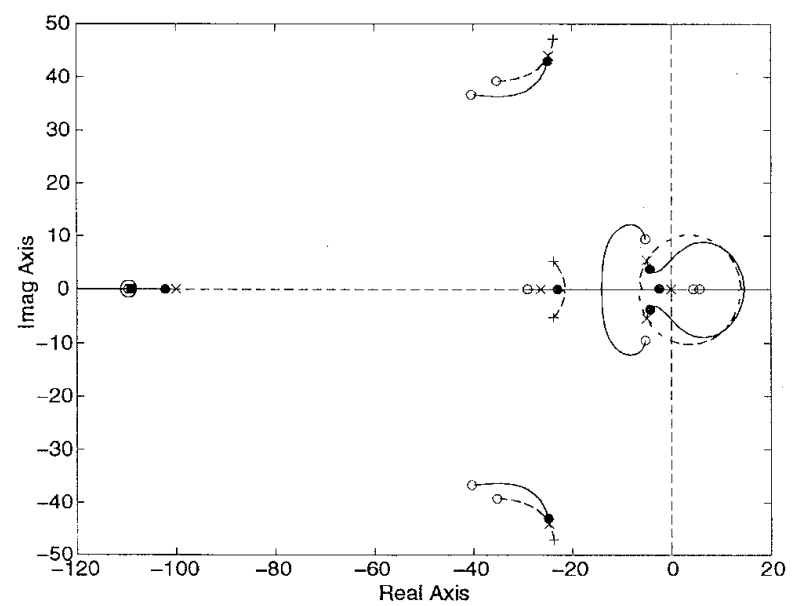

(b)

Fig. 5. Example 2: $\Omega^{2}<k_{2}$. (a) Contour plot of max. real parts of hte closed-loop poles. (b) Root loci.

zeros and no ORHP poles. Hence, we conclude in this case that for all positive values of $k_{1}$ and $k_{3}$ and for sufficiently small negative values of $k_{4}$ and $k_{5},(27)$ is asymptotically stable.

Example 2: In this example, we have $k_{1}=50 \mathrm{~s}^{-1}, k_{2}=$ $1250 \mathrm{~s}^{-2}, k_{3}=100 \mathrm{~s}^{-1}$, and $\Omega=200 \mathrm{r} / \mathrm{min}(20.9 \mathrm{rad}-$ $\mathrm{s}^{-1}$ ), so that $\Omega^{2}<k_{2}$. Fig. 5(a) shows a contour plot of the maximum of the real parts of the closed-loop poles for $k_{4}<0$ and $k_{5}<0$. In particular, $-1000<k_{4}<0$ and $-1000<k_{5}<0$ guarantee that all the closed-loop poles lie in the OLHP. The root loci of $G_{1}(s)$ (dotted lines) and $G_{3}\left(s, k_{4}\right)$ (solid lines) for $k_{4}=-1000 \mathrm{~s}^{-2}$ are given in Fig. 5(b). Finally, choosing $k_{4}=-1000 \mathrm{~s}^{-2}$ and $k_{5}=-200 \mathrm{~s}^{-2}$ yields the closed-loop poles $-109.4,-101.9,-24.4 \pm 43.7 j,-4.9 \pm 2.0 j,-22.9$, and -2.2 (marked "•").

\section{Remark}

For $\Omega^{2}=k_{2}$, it can be shown that there exist no real solutions for $k_{4}$ and $k_{5}$ such that (27) is asymptotically stable. See Section VII on implementation considerations that can overcome this difficulty. 


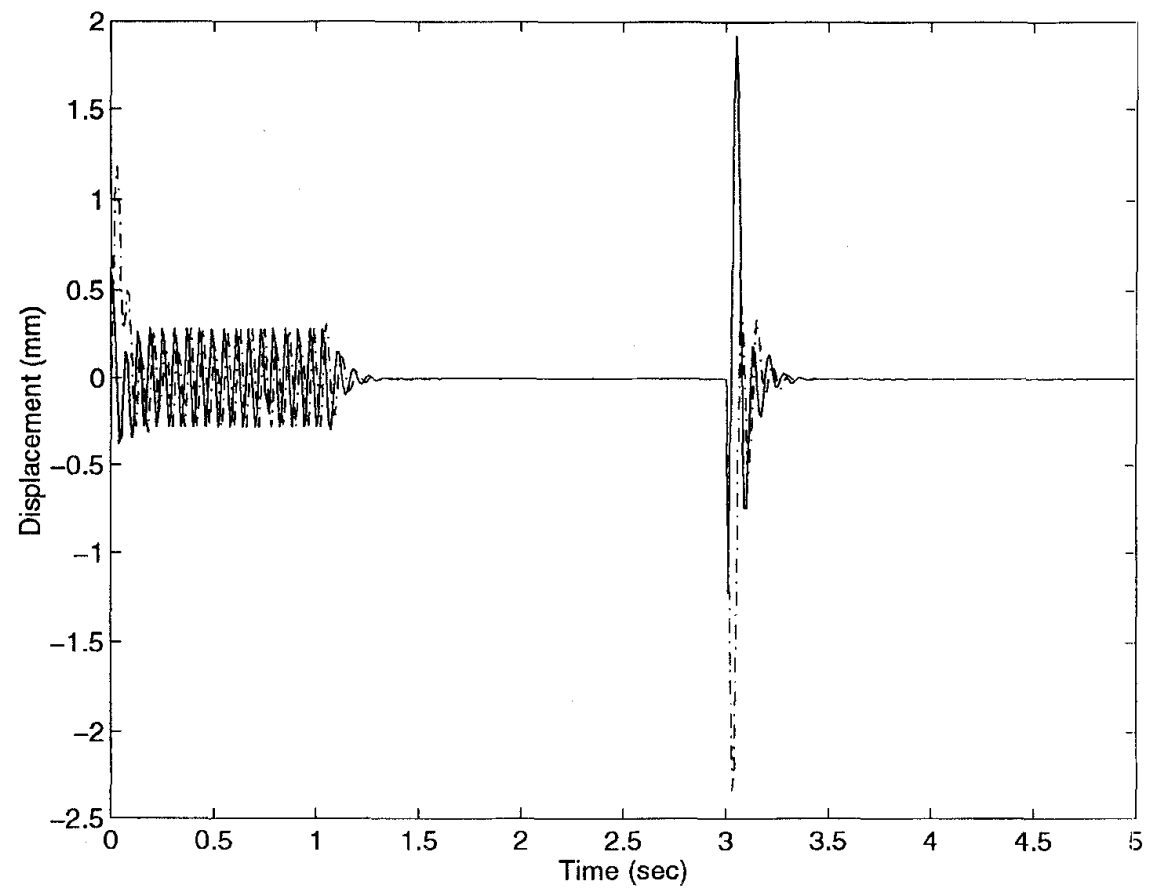

(a)

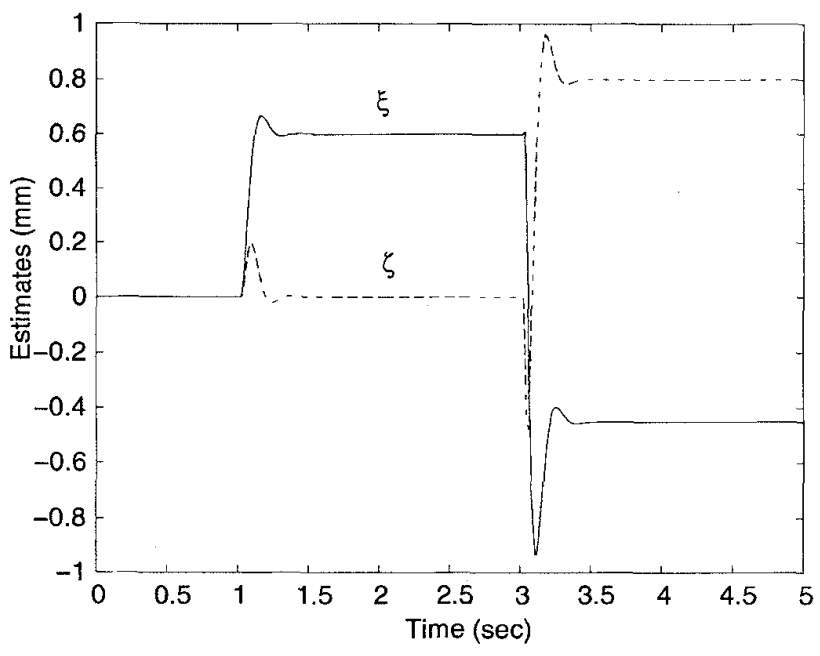

(b)

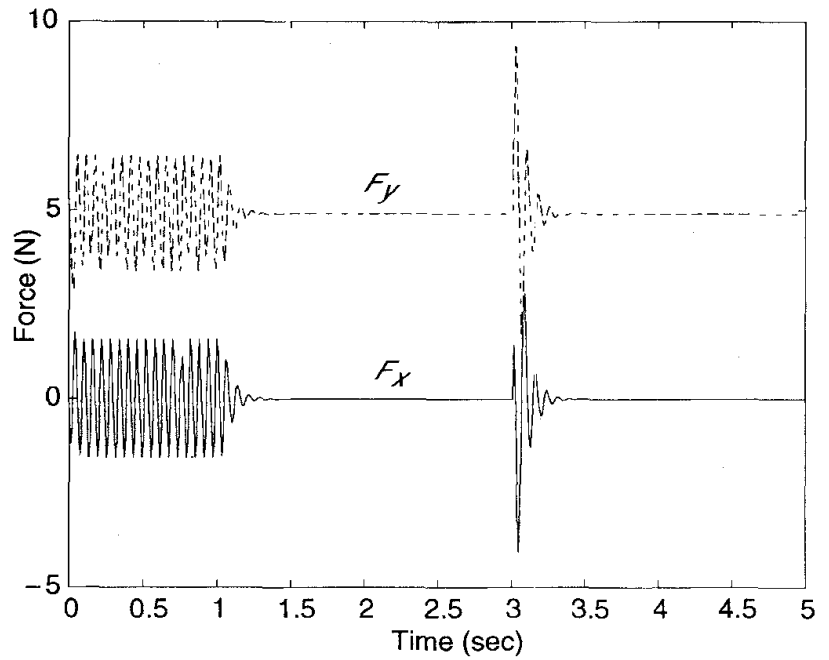

(c)

Fig. 6. Example 3: Static imbalance simulation. (a) $x-$ and $y-$ Displacements of mass center. (b) Parameter estimates: $\hat{\xi}$ and $\hat{\zeta}$. (c) $x-$ and $y-$ acutation forces.

\section{Stability Robustness with Respect to Spin Rate}

Suppose $\left(k_{1}, \cdots, k_{5}\right)$ asymptotically stabilizes $(27)$ as determined according to the previous section. We shall now provide a bound on the spin rate variation $\delta \Omega(t)$ for which the closedloop system remains asymptotically stable. Indeed, consider now the system

$$
\dot{z}=\tilde{A}(\Omega+\delta \Omega(t)) z .
$$

It can be shown that $\tilde{A}(\Omega+\delta \Omega(t))=\tilde{A}(\Omega)+\delta \Omega(t) M$, where $M$ is a constant matrix with induced Euclidean norm $\|M\|=\sqrt{1+k_{1}^{2}}$. Since $\tilde{A}(\Omega)$ is Hurwitz, there exists a positive-definite matrix $P_{\Omega} \in \Re^{8 \times 8}$ such that $\tilde{A}^{\mathrm{T}}(\Omega) P_{\Omega}+$ $P_{\Omega} \tilde{A}(\Omega)=-I$. Consider the Lyapunov function candidate $V_{\Omega}(z) \triangleq z^{\mathrm{T}} P_{\Omega} z$ which is obviously $C^{1}$ and positive definite on $\Re^{8}$. Then, along the trajectories of (29), we have $\dot{V}_{\Omega}=$ $-\|z\|^{2}+2 \delta \Omega(t) z^{\mathrm{T}} P_{\Omega} M z$. For $\delta \Omega(t)$ verifying

$$
|\delta \Omega(t)| \leq \frac{1}{4\left\|P_{\Omega}\right\| \sqrt{1+k_{1}^{2}}}
$$

we thus have $\dot{V}_{\Omega} \leq \frac{1}{2}\|z\|^{2}$, for all $z \in \Re^{8}$, which guarantees uniform asymptotic stability of (29) [9]. This shows that $\left(k_{1}, \cdots, k_{5}\right)$ achieves robust asymptotic stability of the closed 


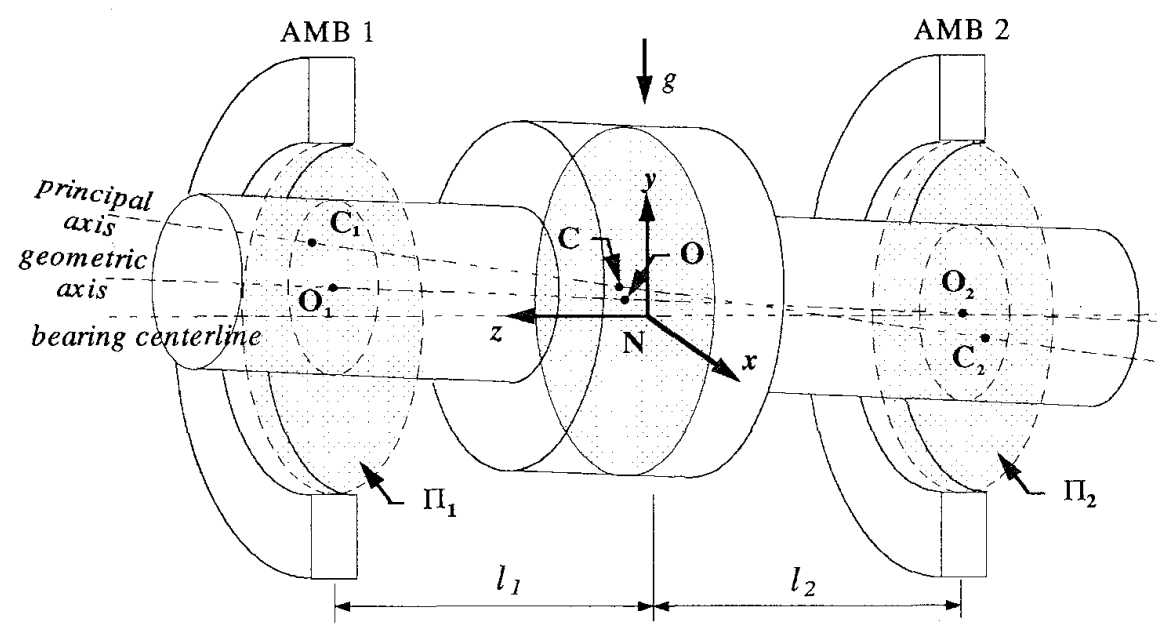

Fig. 7. Dynamically unbalanced rotor supported by two AMB's.

loop for small variation in spin rate. Note however that (30) is only a sufficient condition and, hence, may provide a conservative estimate of stability robustness.

\section{Simulation Example--Static Balancing}

Example 3: We now demonstrate the performance of the AAC control law by means of a simulation example. Consider the planar motion of a rigid circular disk having the following characteristics: nominal mass $m=0.5 \mathrm{~kg}$, radius $r=0.3 \mathrm{~m}$, and hence nominal moment of inertia $J=0.0225 \mathrm{~kg}-\mathrm{m}^{2}$. The AMB has the stiffness coefficients $K_{h}=200 \mathrm{~N} / \mathrm{m}$ and $K_{i}=5$ $\mathrm{N} / \mathrm{A}$. The spin rate is fixed at $1000 \mathrm{r} / \mathrm{min}\left(104.7 \mathrm{rad}-\mathrm{s}^{-1}\right)$ and we consider the AAC controller settings given in Example 1.

The initial imbalance of the disk is such that $(\xi, \zeta)=$ $(0.6,0) \mathrm{mm}$. Between $t=0$ and $t=1 \mathrm{~s}$, parameter adaptation is disabled by maintaining $\hat{\xi}=\hat{\zeta} \equiv 0$. During this first second, the control (18) and (19) simply emulates an elastic suspension. As shown in Fig. 6(a), as parameter adaptation is enabled at $t=1 \mathrm{~s}$., the mass center displacements in both axes are attenuated within $0.2 \mathrm{~s}$.

At $t=3 \mathrm{~s}$, the imbalance changes to $(\xi, \zeta)=(-0.45,0.8)$ $\mathrm{mm}$. The controller is able to adapt and achieves rotation about the mass center within $0.2 \mathrm{~s}$. The adaptability of the control law is apparent in Fig. 8(b), where it can be seen that $\hat{\xi}$ and $\hat{\zeta}$ converge to the actual values. Fig. $6(\mathrm{c})$ confirms the observation made in Section III that when rotation about the mass center is achieved, synchronous components of the actuation forces are zero and, hence, synchronous vibration attenuation is also achieved. Note that $F_{y}$ converges to the weight of the rotor.

\section{REMARKS ABOUT IMPLEMENTATION}

We have seen that in order to achieve closed-loop stability, we need to examine the relative values of $\Omega^{2}$ and $k_{2}$. It can be seen from (22) and (23) that $k_{2}$ plays the role of a stiffness coefficient of an elastic suspension, thus giving rise to the critical speed $\Omega_{c}=\sqrt{k_{2}}$. The results of the previous section show that convergence of AAC can be achieved both above and below critical speed, that is $\Omega>\Omega_{\mathrm{c}}$ and $\Omega<\Omega_{\mathrm{c}}$. However, it can be shown that at critical speed, that is, $\Omega=\Omega_{c}$, no values of $k_{4}$ and $k_{5}$ can be found to obtain asymptotic stability of the AAC closed loop. Nevertheless, this does not constitute a real disadvantage of AAC. In theory, $k_{2}$ can be chosen so that the critical speed is outside of the desired operating range of $\Omega$. In practice, the desired stiffness may require that $\Omega_{c}$ be inside the operating range of $\Omega$. This apparent difficulty can easily be overcome by allowing the adaptation algorithm to converge at spin rates different from the critical speed, then disabling the adaptation and using the fixed estimates while crossing the critical speed.

A more subtle issue at hand is that asymptotic estimation of the imbalance parameters $(\xi, \zeta)$ allows the control objective, that is, synchronous vibration attenuation, to be achieved even under changing spin rate. Indeed, as seen in Section V-D, $(\hat{\xi}, \hat{\zeta})$ can be made to converge for sufficiently small variation of spin rate about any design spin rate $\Omega$. Since imbalance is independent of the spin rate, adaptation can then be halted and the spin rate can be allowed to vary according to the function of the rotor, say, in spin-ups or near the critical speed, without affecting closed-loop asymptotic stability of rotor mass center motion. This property can be verified by substituting $\tilde{\xi}=0$ and $\tilde{\zeta}=0$ in $(22)$ and (23) which then recovers (10) and (11). In addition, if the synchronous vibration level exceeds the prescribed tolerance due to an imbalance change, then the spin rate can be stabilized at some constant value and adaptation reactivated at that spin rate. In this aspect, $\mathrm{AAC}$ presents an advantage over AFFC methods, where model dependence on spin rate requires continual adaptation when the spin rate changes [11], [18].

\section{ADAPTIVE AUTOCENTERING CONTROL-DYNAMIC IMBALANCE}

\section{A. Formulation}

In this section consider the rigid rotor of Fig. 7, where it is shown supported by a pair of AMB's. Let $\Pi_{1}$ and $\Pi_{2}$ denote the action planes of the respective AMB's. The 


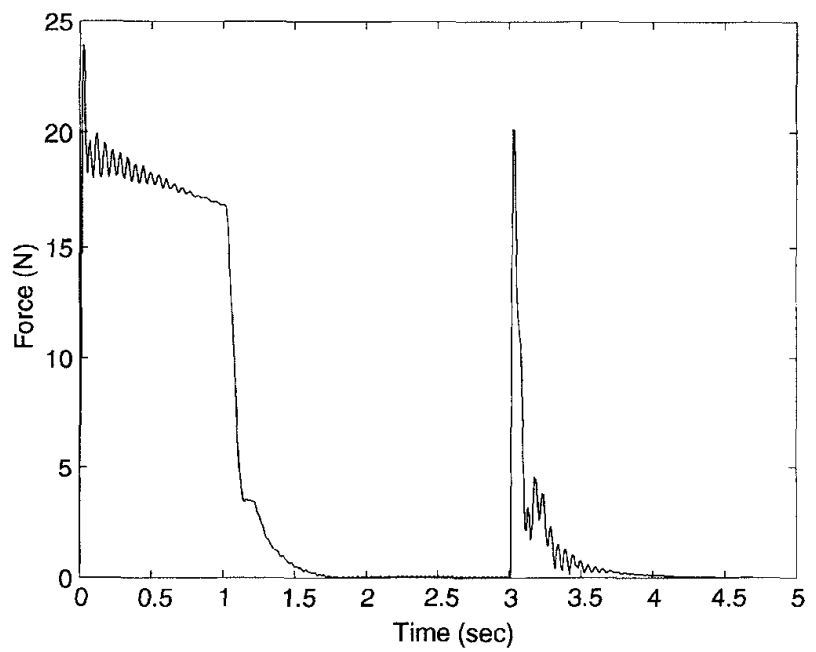

(a)

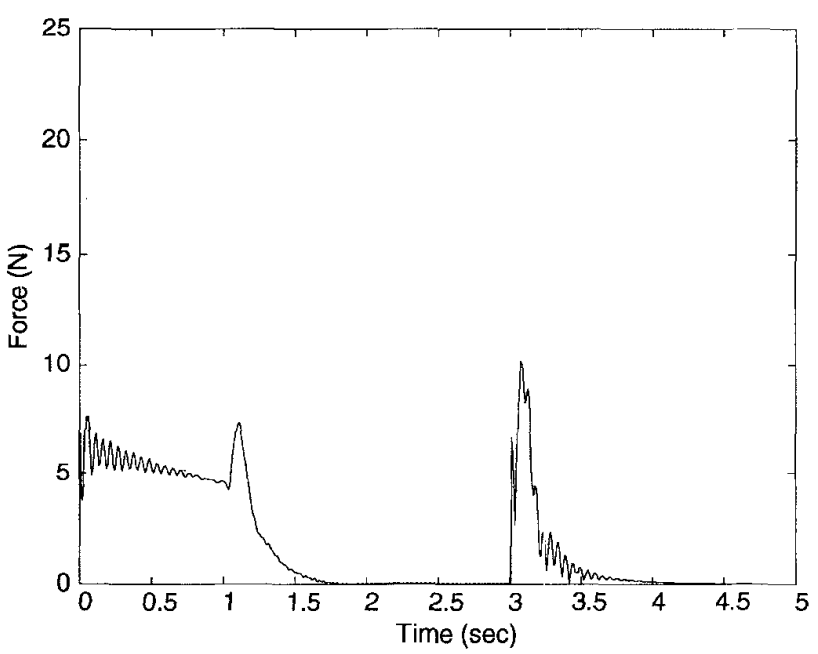

(b)

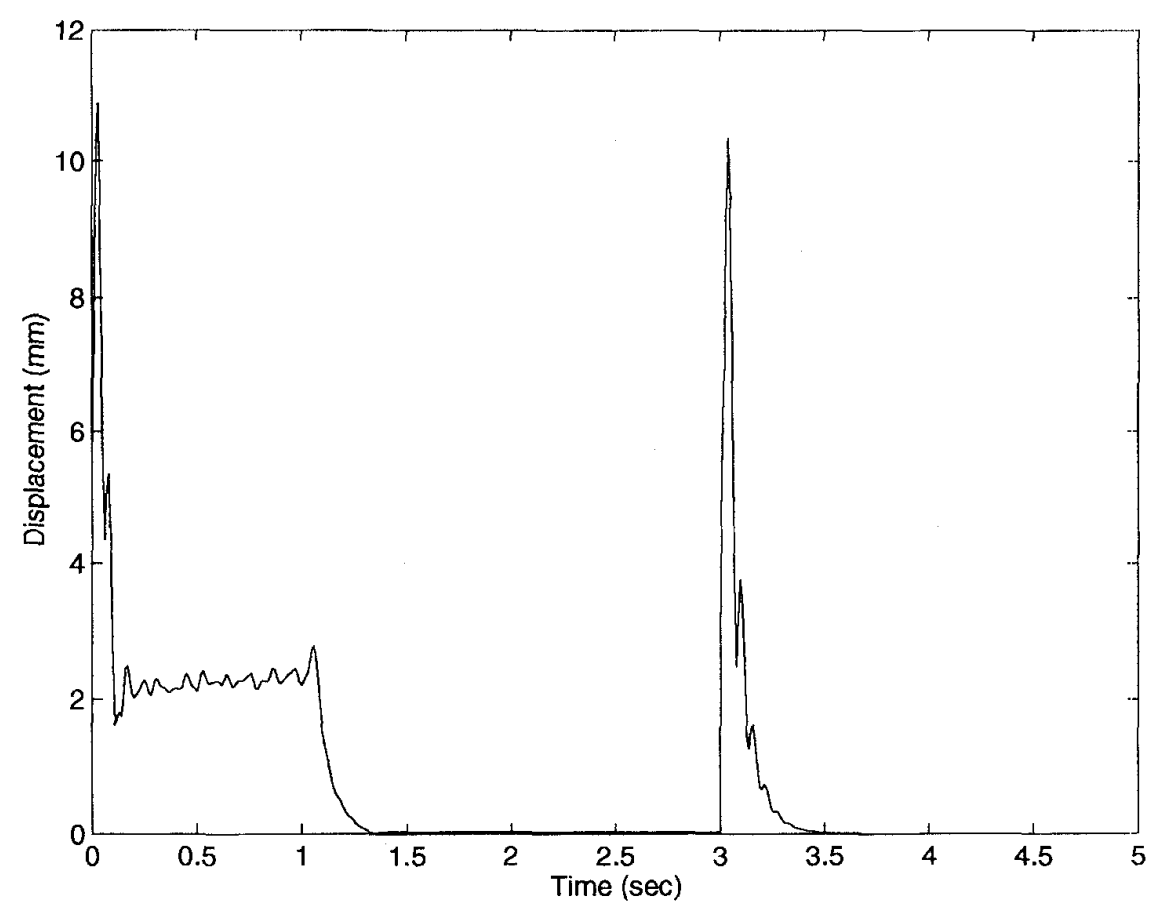

(c)

Fig. 8. Adaptive autocentering example: dynamic imbalance. (a) Amplitudes of synchronous transmitted force ( $F_{x_{1}}$ ). (b) Amplitudes of synchronous transmitted force $\left(F_{x_{2}}\right)$. (c) Amplitude of mass center displacement.

geometric axis of the rotor intersects $\Pi_{1}$ and $\Pi_{2}$ at $\mathrm{O}_{1}$ and $\mathrm{O}_{2}$, respectively, whereas the principal axis (passing through the mass center $C$ ) intersects $\Pi_{1}$ and $\Pi_{2}$ at $\mathrm{C}_{1}$ and $\mathrm{C}_{2}$. Choosing the inertial coordinate system determined by the fixed point $\mathrm{N}$ and the Cartesian coordinates $(x, y, z)$, where $\mathrm{N} z$ coincides with the bearing centerline, the points $\mathrm{O}_{1}$ and $\mathrm{C}_{1}$ (respectively, $\mathrm{O}_{2}$ and $\mathrm{C}_{2}$ ) can be located in $\Pi_{1}$ (respectively, $\Pi_{2}$ ) by their $(x, y)$-coordinates $\left(X_{1}, Y_{1}\right)$ and $\left(x_{1}, y_{1}\right)$ [respectively, $\left(X_{2}, Y_{2}\right)$ and $\left.\left(x_{2}, y_{2}\right)\right]$. Assuming small angular deflections about the bearing centerline, the kinematics of $\mathrm{O}_{1}$ and $\mathrm{C}_{1}$ (respectively, $\mathrm{O}_{2}$ and $\mathrm{C}_{2}$ ) are related by

$$
\begin{aligned}
X_{n} & =x_{n}-\xi_{n} \cos \Omega t+\zeta_{n} \sin \Omega t \\
Y_{n} & =y_{n}-\xi_{n} \sin \Omega t-\zeta_{n} \cos \Omega t
\end{aligned}
$$

for $n \in\{1,2\}$, where $\left(\xi_{n}, \zeta_{n}\right)$ are Cartesian coordinates of $\mathrm{C}_{n}$ relative to $\mathrm{O}_{n}$ in a rotating frame, and $\Omega$ is the rotor spin rate that is assumed constant. The variables $\left(\xi_{n}, \zeta_{n}\right)$ are uniquely determined by the dynamic imbalance of the rotor (see [2]) and hence are unknown parameters playing the same role as $(\xi, \zeta)$ in the planar case. Moreover, asymptotically stabilizing 
the motions of $\mathrm{C}_{1}$ and $\mathrm{C}_{2}$ will result in rotation about the principal axis of inertia, and thus in attenuation of synchronous transmitted forces at both bearings. With this observation, we now extend the AAC control law for a rigid rotor with unknown dynamic imbalance by specializing (12)-(19) to the respective balancing planes as given by

$$
\begin{aligned}
& \hat{x}_{n} \triangleq X_{n}+\hat{\xi}_{n} \cos \Omega t-\hat{\zeta}_{n} \sin \Omega t \\
& \hat{y}_{n} \triangleq Y_{n}+\hat{\xi}_{n} \sin \Omega t+\hat{\zeta}_{n} \cos \Omega t \\
& \hat{\dot{x}}_{n} \triangleq \dot{X}_{n}-\hat{\xi}_{n} \Omega \sin \Omega t-\hat{\zeta}_{n} \Omega \cos \Omega t \\
& \hat{\dot{y}}_{n} \triangleq \dot{Y}_{n}+\hat{\xi}_{n} \Omega \cos \Omega t-\hat{\zeta}_{n} \Omega \sin \Omega t \\
& \ddot{\hat{\xi}}_{n}=-k_{3} \dot{\hat{\xi}}_{n}-k_{4}\left(\hat{x}_{n} \cos \Omega t+\hat{y}_{n} \sin \Omega t\right) \\
& \ddot{\hat{\zeta}}_{n}=-k_{3} \dot{\hat{\zeta}}_{n}-k_{5}\left(-\hat{x}_{n} \sin \Omega t+\hat{y}_{n} \cos \Omega t\right) \\
& \dot{i}_{x_{n}}=-\frac{K_{h}}{K_{i}} X_{n}-\frac{m}{K_{i}}\left(k_{1} \hat{\dot{x}}_{n}+k_{2} \hat{x}_{n}\right) \\
& i_{y_{n}}=-\frac{K_{h}}{K_{i}} Y_{n}-\frac{m}{K_{i}}\left(k_{1} \hat{\dot{y}}_{n}+k_{2} \hat{y}_{n}\right)+\frac{m g}{2 K_{i}}
\end{aligned}
$$

for $n \in\{1,2\}$, where $k_{1}, k_{2}, k_{3}, k_{4}$ and $k_{5}$ are constants to be chosen. Based on the earlier results in the single plane case, we conjecture that in each balancing plane $\Pi_{n}, n \in$ $\{1,2\},\left(\hat{\xi}_{n}, \hat{\zeta}_{n}\right)$ are asymptotic estimates of $\left(\xi_{n}, \zeta_{n}\right)$, and the closed-loop motion of $\mathrm{C}_{n}$ can be asymptotically stabilized by choosing $k_{1}, \cdots, k_{5}$ appropriately. This conjecture appears to be reasonable for small angular deflections of the rotor. Moreover, we shall see in the following simulation example that it is valid.

\section{B. Simulation Example}

Here, we consider a $0.5 \mathrm{~kg}$ rotor with a radius of $0.3 \mathrm{~m}$ and width of $0.2 \mathrm{~m}$. The distance $L$ between the two AMB's is 0.4 In. The dynamic imbalance of the rotor is such that the inertia matrix, resolved in a body coordinate system where the 3-axis coincides with the rotor geometric axis and the 1- and 2-axes are arbitrary, is given by

$$
J=\left[\begin{array}{ccc}
0.0147 & 0.0000 & 0.0004 \\
0.0000 & 0.0147 & -0.0004 \\
0.0004 & -0.0004 & 0.0229
\end{array}\right]
$$

and the body coordinates of the mass center are $(3.7,3.7,0)$ $\mathrm{mm}$. The same control parameters as in Example 3, Section VI, are used, and the spin rate is fixed at $1080 \mathrm{r} / \mathrm{min}(113.1$ rad-s $\left.{ }^{-2}\right)$. Although small angles are assumed in the previous subsection to illustrate how AAC can be extended to dynamic balancing, the simulation routine used in this example is comprised of the full-order nonlinear equations of motion of the rotor. The only small angle approximation made here is the use of the linear radial AMB model (3) and (4). A linear thrust bearing model is also incorporated to ensure stability of the motion in the $z$-direction.

Fig. 8(a) and (b) shows that when two-plane AAC control is enabled at $t=1 \mathrm{~s}$, the amplitudes of the actuation forces at both AMB are attenuated within $1 \mathrm{~s}$. Fig. 8(c) shows that motion of the mass center is also attenuated. Fig. 9(a) and (b) shows the convergence of the estimates $\left(\hat{\xi}_{1}, \hat{\zeta}_{1}\right)$ and $\left(\hat{\xi}_{2}, \hat{\zeta}_{2}\right)$, respectively. In particular, using the steady-state values of these estimates between $t=2 \mathrm{~s}$ and $t=3 \mathrm{~s}$, the estimated

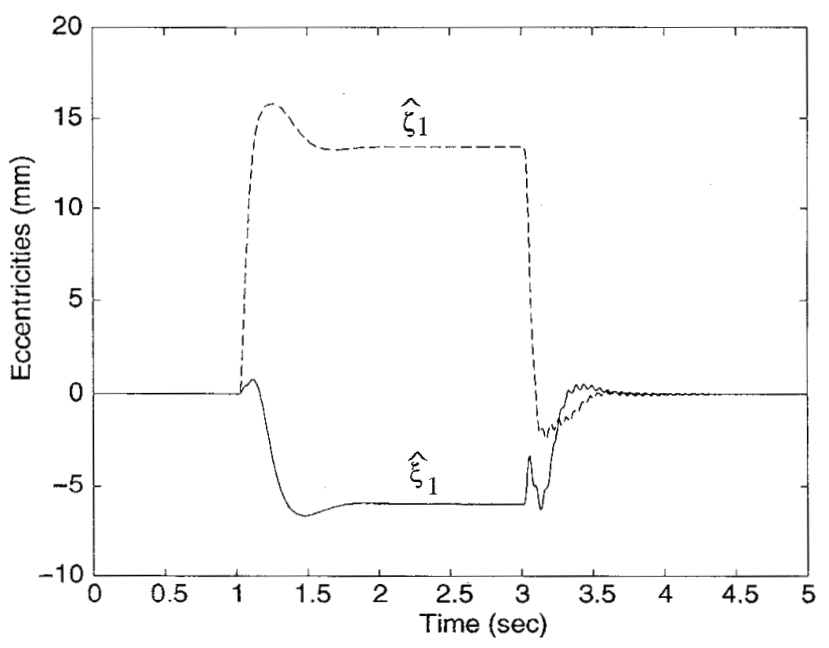

(a)

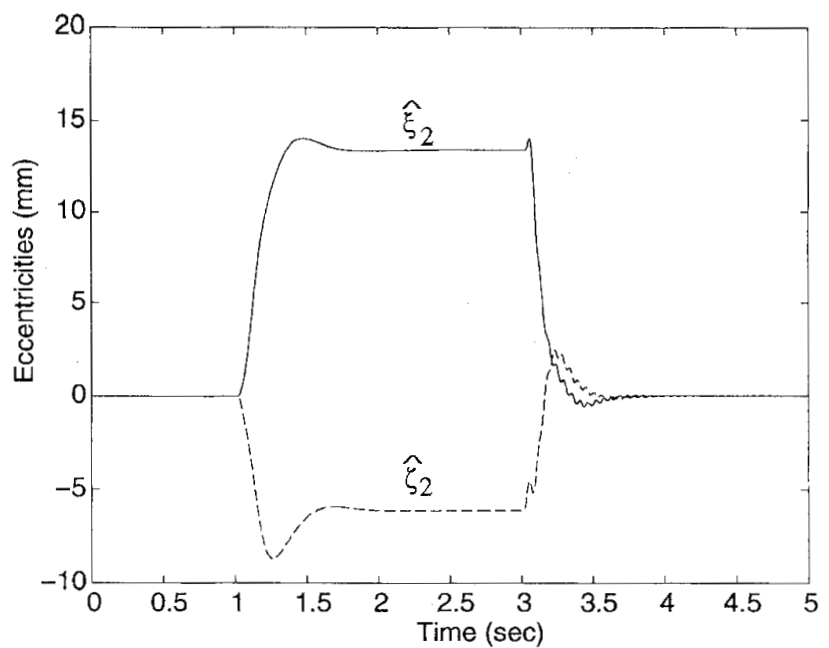

(b)

Fig. 9. Adaptive autocentering example: dynamic imbalance. (a) Estimates $\hat{\xi}_{1}$ and $\hat{\zeta}_{1}$. (b) Estimates $\hat{\xi}_{2}$ and $\hat{\zeta}_{2}$.

coordinates of $\mathrm{C}_{1}$ and $\mathrm{C}_{2}$ relative to the chosen body frame are

$$
\begin{aligned}
\mathrm{OC}_{1} & =\left[\begin{array}{c}
\hat{\xi}_{1} \\
\hat{\zeta}_{1} \\
-\frac{L}{2}
\end{array}\right]=\left[\begin{array}{c}
-0.6 \\
13.4 \\
-200
\end{array}\right] \mathrm{mm} \\
\mathrm{OC}_{2} & =\left[\begin{array}{c}
\hat{\xi}_{2} \\
\hat{\zeta}_{2} \\
\frac{L}{2}
\end{array}\right]=\left[\begin{array}{c}
3.4 \\
-0.6 \\
200
\end{array}\right] \mathrm{mm} .
\end{aligned}
$$

It can then be verified that $\mathrm{C}_{1} \mathrm{C}_{2}$ passes through $\mathrm{C}$ and is an eigenvector of $J$. Finally, at $t=3 \mathrm{~s}$, the imbalance is artificially removed, resulting in momentary vibration during readaptation.

\section{CONCluding Remarks}

We have derived the adaptive autocentering control law for an active magnetic bearing supporting a rigid rotor with unknown mass imbalance. The objective of AAC is to asymptotically stabilize the motion of the mass center and the 
principal axis of inertia. We showed that this control objective is equivalent to the attenuation of transmitted forces at the AMB. To achieve its objective, AAC incorporates an adaptation algorithm that performs on-line identification of the mass center location $\mathrm{C}$ for a planar rotor (Fig. 2), and of the principal axis of inertia determined by the points $C_{1}$ and $\mathrm{C}_{2}$ (Fig. 7) for a three-dimensional rotor. Using root locus arguments, an existence proof of control parameter values that render the closed-loop system asymptotically stable was given in the case of a statically unbalanced rotor in planar motion with constant spin rate. In addition, a two-plane implementation of AAC control was shown in simulation to correctly identify and asymptotically stabilize the mass center and principal axis of inertia of a dynamically unbalanced rotor in six degree-of-freedom motion.

As pointed out in Section VII, since the algorithm identifies physical imbalance parameters which, for a rigid rotor, are independent of the spin rate, adaptation can be halted once parameter convergence is achieved. This unique feature of AAC thus allows the control objective, that is, transmitted force attenuation, to be achieved at arbitrary spin rate by yielding closed-loop dynamics of the form (10) and (11), even during spin-ups or while crossing critical speed.

The above results, however, are valid for rigid rotors only and cannot be immediately applied in high-speed industrial applications where rotors exhibit flexible behavior. Our ultimate objective is therefore to extend the concept of AAC to flexible rotors with unknown imbalance. Modifications to provide robustness with respect to AMB nonlinearity and model uncertainty, as well as rotor spin-up, are also of interest.

\section{REFERENCES}

[1] P. Bovik and C. Hogfors, "Autobalancing of rotors," J. Sound Vibration, vol. 111, no. 3, pp. 429.440, 1986

[2] M. S. Darlow, Balancing of High-Speed Machinery. New York: Springer-Verlag, 1989.

[3] G. F. Franklin, J. D. Powell, and A. Emami-Naeini, Feedback Control of Dynamic Systems, 3rd ed. New York: Addison-Wesley, 1994.

[4] J. K. Hale, Ordinary Differential Equations, 2nd ed. Huntington, NY: Krieger, 1980.

[5] T. Higuchi, T. Mizumo, and M. Tsukamoto, "Digital control system for magnetic bearings with automatic balancing," in Proc. 2nd Int. Symp. Magn. Bearings, July 12-14, 1990, Tokyo, Japan, pp. 27-32.

[6] R. R. Humphris, R. D. Kelm, D. W. Lewis, and P. E. Allaire, "Effect of control algorithms on magnetic journal bearing properties," Trans Amer. Soc. Mech. Eng., vol. 108, pp. 624-632, Oct. 1986.

[7] J. Imblach, B. J. Blair, and P. E. Allaire, "Measured and predicted force and stiffness characteristics of industrial magnetic bearings," Trans. Amer. Soc. Mech. Eng., vol. 113, pp. 784-788, Oct. 1991.

[8] J. Inoue, S. Hayashi, and Y. Araki, "On the self-synchronization of mechanical vibrators," Bull. Japan Soc. Mech. Eng., vol. 10, no. 41, pp 755-762, 1967.

[9] H. K. Khalil, Nonlinear Systems. New York: Macmillan, 1992.

[10] C. R. Knospe, R. W. Hope, S. J. Fedigan, and R. D. Williams, "Adaptive on-line rotor balancing using digital control," in Proc. MAC '93 Magn. Bearings, Magn. Drives, and Dry Gas Seal Conf., July 1993, pp. 156-164.

[11] C. R. Knospe, R. W. Hope, S. J. Fedigan, and R. D. Williams, "New results in the control of rotor synchronous vibration," Center for Magn. Bearings, Univ. Virginia, Charlottesville, VA, Internal Rep., 1994.

[12] R. Larsonneur and R. Herzog, "Feedforward compensation of unbalance New results and application experiences," in Proc. IUTAM Symp. Active Contr. Vibration, Sept. 1994, Bath, U.K.

[13] K.-Y. Lum, S. P. Bhat, V. T. Coppola, and D. S. Bernstein, "Adaptive virtual autobalancing for a magnetic rotor with unknown mass imbalance, I. Static balancing," in Proc. 1995 Design Eng. Tech.
Conf-Acoust, Vibrations, and Rotating Machines, vol. 3, part B, vol. DE 84-2, Boston, MA, Sept. 1995, pp. 1419-1425.

[14] _ " Adaptive virtual autobalancing for a magnetic rotor with unknown mass imbalance, II. Dynamic balancing," in Proc. 1995 Design Eng. Tech. Conf.-Acoust., Vibrations, and Rotating Machines, vol. 3, part B, vol. DE 84-2, Boston, MA, Sept. 1995, pp. 1427-1434.

[15] E. H. Masien, P. E. Allaire, R. R. Humphris, M. Noh, and C. K. Sotore, "Magnetic bearing design for reduced powe." consumption," 1995.

[16] F. Matsumura, M. Fujita, and K. Okawa, "Modeling and control of magnetic bearing system achieving a rotation around the axis of inertia," in Proc. 2nd Int. Symp. Magn. Bearings, July 12-14, 1990, Tokyo, Japan, pp. $273-280$.

[17] T. Mizuno and T. Higuchi, "Design of magnetic bearing controllers based on disturbance estimation," in Proc. 2nd Int. Symp. Magn. Bearing, July 12-14, 1990, Tokyo, Japan, pp. 281-238.

[18] B. Shafai, S. Beale, P. LaRocca, and E. Cusson, "Magnetic bearing control systems and adaptive forced balancing," IEEE Contr. Syst. Mag., vol. 14, no. 2, pp. 4-13, 1994.

[19] R. Siegwart, "Design and application of active magnetic bearings (AMB) for vibration control," Von Karman Inst. Fluid Dynamics Lecture Series 1992-06, Vibration and Rotor Dynamics, Sept. 21-25, 1992.

[20] G. Schweitzer, "Stabilization of self-excited rotor vibrations by an active damper," in Dynamics of Rotors, F. I. Niordson, Ed. New York: Springer-Verlag, 1975

[21] E. L. Thearle, "Automatic dynamic balancers, Part 2-Ring, pendulum and ba11 balancers," Machine Design, vol. 23, pp. 103-152, 1950.

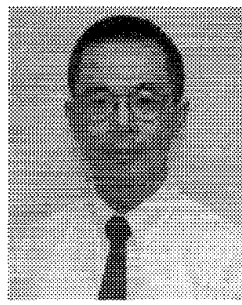

Kai-Yew Lum received the Diplôme d'Ingénieur degree from the Ecole Nationale Supérieure d'Ingénieurs Electriciensi de Grenoble, France, in 1988 , and the M.S. degree in aerospace engineering from the University of Michigan, Ann Arbor, in 1995. He is currently a Ph.D. candidate with the Department of Aerospace Engineering at the University of Michigan.

His current research interests are nonlinear control of mechanical systems and geometric theory in mechanics.

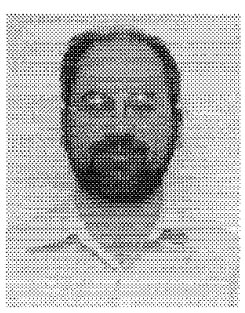

Vincent T. Coppola received the Ph.D. degree in theoretical and applied mechanics from Cornell University, Ithaca, NY, in 1989.

Before coming to Michigan in 1991, he was an ASEE Postdoctoral Fellow at the Naval Resarch Laboratory. He is currently an Assistant Professor of Aerospace Engineering at the University of Michigan, Ann Arbor.He teaches courses in flight mechanics, orbital mechanics, intermediate and advanced dynamics, and nonlinear dynamical systems. His research interests include nonlinear vibrations, perturbation methods, and the dynamics of rotating systems.

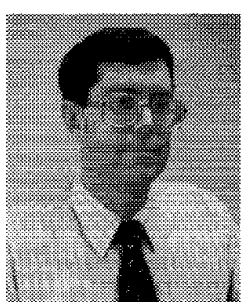

Dennis S. Bernstein (M'82) received the Ph.D. degree in coumpter, information, and control engineering from the University of Michigan, Ann Arbor, in 1982

From 1982 to 84 he was employed by MIT Lincoln Laboratory, and from 1984 to 1991 he worked for the Harris Corporation, Melbourne, FL. He is an Associate Professor in the Department of Aerospace Engineering at the University of Michigan, where he has taught courses on control of flexible structures, optimal control, and multivariable feedback control theory. His research interests include linear and nonlinear control, experimental control of vibrations in solids, liquids, and gases with application to flexible structures, noise suppression, combustion, and slosh, as well as control of rigid body motion with application to rotating spacecraft and unbalanced rotating machinery. 